\title{
Influence of Temperature on the Steady State and Transient Luminance of an OLED Display
}

\author{
Frédérique Chesterman, Bastian Piepers, Tom Kimpe, Patrick De Visschere, and Kristiaan Neyts
}

\begin{abstract}
In this paper, the influence of temperature on the luminance of an organic light-emitting device (OLED) display is investigated. Luminance, temperature, and power measurements are executed on a 55-in white-red-green-blue active-matrix OLED display with a resolution of $1920 \times 1080$ and an oxide-thin-filmtransistor (TFT) backplane, under a controlled, static temperature environment. The measurements indicate a strong influence of temperature on the luminance of the display, resulting from the temperature dependence of both the TFT and the OLED. The influence of temperature on the luminance of an OLED display is also investigated in a dynamic context. Measurements show that temperature changes resulting from losses in the display have an important influence on the luminance stability of the display. The measurements linking luminance and temperature in a static temperature environment allow estimating the change in luminance in a dynamic context. Finally, this paper presents the results of a number of experiments that were set up to show scenarios in which the temperature dependence of the display's luminance has a direct negative impact on the picture quality of the display. The results of this work show that the thermal behavior of an OLED display must be taken into account when working towards a high-performing OLED display.
\end{abstract}

Index Terms-Luminance, OLED display, OLED efficiency, power consumption, temperature, transient.

\section{INTRODUCTION}

$\mathbf{T}$ WENTY-NINE years have passed since the discovery of the organic light-emitting device (OLED) working principle [1]. The potential of OLED technology for display applications was identified many years ago, but it is only recently that mass production of OLED displays really took off. First there was mass production of small-sized OLED displays for mobile applications such as smartphones and later tablets. More recently, also the production of large-sized OLED panels (55 inch diagonal and more) for $\mathrm{TV}$ applications reached relatively large volumes. It is only in 2016 that OLED technology made the

Manuscript received May 12, 2016; revised August 17, 2016; accepted August 23, 2016. Date of publication August 31, 2016; date of current version October 11, 2016. This work was supported by the IWT (Institute for the Promotion of Innovation by Science and Technology in Flanders) in the context of the Baekeland Grant "Applicability of OLED technology in medical displays," 130300 .

F. Chesterman is with the Healthcare Division, Barco NV, Oost-Vlaanderen, Gent, Belgium (e-mail: frederique.chesterman@barco.com).

B. Piepers is with the Healthcare Division, Barco NV, West-Vlaanderen, Kortrijk, Belgium (e-mail: bastian.piepers@barco.com).

T. Kimpe is with the Medical Imaging Division, Barco NV, West-Vlaanderen, Kortrijk, Belgium (e-mail: tom.kimpe@barco.com).

P. D. Visschere and K. Neyts are with the Department of Electronics and Information Systems, Ghent University, Oost-Vlaanderen, Gent, Belgium (e-mail:pdv@elis.ugent.be; kneyts@elis.ugent.be).

Color versions of one or more of the figures are available online at http://ieeexplore.ieee.org.

Digital Object Identifier 10.1109/JDT.2016.2605038 step to more general display applications such as medium-sized monitors and screens for laptops.

Notwithstanding the significance of these steps forward, a few considerations must be made when employing OLED technology for demanding applications such as medical displays, where it is crucial to maintain constant picture quality under varying circumstances and throughout the lifetime of the display [2], [3]. Indeed, it was found previously that the temperature distribution across an OLED display is non-uniform, time-varying and a function of the image content [4]-[11]. Considering on the one hand the relationship between temperature and the intensity of the light emission of an OLED [12]-[17] and on the other hand the influence of temperature on the TFT characteristic [18]-[21], the thermal behavior of an OLED display has a direct impact on the picture quality. These findings indicate that a thorough understanding of the link between temperature and the light emission of an OLED display is crucial to understand the display's shortcomings and eventually realize OLED displays that are suitable for the medical display market.

In response to this need, this paper investigates the influence of temperature on the light emission of an OLED display, through measurements on a 55 inch white-red-green-blue (WRGB) active-matrix OLED display with a resolution of $1920 \times 1080$ and an oxide-TFT backplane, hereafter referred to as 'OLED display'. In Section II, more details on the technologies used in this display will be provided. The results of luminance and power measurements under static temperature conditions are presented in Section III. The measurements are used to calculate the temperature dependency of the TFT characteristic and the OLED efficiency. The results of luminance and power measurements under dynamic temperature conditions are presented in Section IV. It is shown that the measurements linking temperature and luminance under static temperature conditions can also be used to estimate luminance changes resulting from changes in temperature. Finally, the importance of the identified relationship between temperature and light emission is illustrated through a number of experiments in which the thermal behavior of an OLED display is directly causing degraded picture quality. These results are presented in Section V.

The conclusions stated in this paper are resulting from measurements on a 55 inch WRGB OLED display with oxide-TFT backplane. It is uncertain whether similar conclusions can be drawn for other types of OLED displays, with different technologies (e.g., red-green-blue (RGB) OLED display technology or low-temperature-poly-silicon (LTPS) based backplanes) or with different dimensions. 


\section{DEVICE Under Test}

The OLED display on which all measurements presented in the remainder of this paper are performed is using the WRGB OLED technology [4] and an oxide-TFT backplane. The display is a 2014 model. WRGB OLED technology is currently most mature for large-sized OLED displays. In this technology, each pixel comprises four sub-pixels based on white OLEDs. Colors are obtained by placing filters in front of three sub-pixels. A fourth sub-pixel has no filter in order to increase the white efficiency. Oxide-TFT is a relatively new but promising technology for TFT backplanes.

It was found experimentally that for the white point of the display [DDL $(255,255,255)]$, the white, blue and red sub-pixels are used [4]. DDLs $(255,0,0),(0,255,0)$ and $(0,0,255)$ are respectively using only the red, green and blue sub-pixels. As the quadruplet $(W, R, G, B)$ representing the driving of each of the four sub-pixels is a transformation of the DDL triplet $\left(D D L_{R}, D D L_{G}, D D L_{B}\right)$, there must also exist a triplet for which only the white sub-pixel is used. This triplet was determined experimentally as $(252,255,215)$. As we are regularly referring to two different types of white light, the following naming will be maintained throughout this paper. "the display's nominal white" refers to the light obtained when driving the display at DDL $(255,255,255)$. "the light emitted by the white sub-pixel" corresponds to DDL $(252,255,215)$.

\section{TEMPERATURE DEPENDENCY}

In this section, the luminance and power consumption of the OLED display are investigated as a function of temperature.

The display is placed inside a temperature chamber and the ambient temperature $T_{\text {amb }}$ is swept from $-5{ }^{\circ} \mathrm{C}$ to $45^{\circ} \mathrm{C}$ with a step of $5{ }^{\circ} \mathrm{C}$. The ambient temperature is maintained at each temperature level for two hours. During each two hour time slot, a full screen uniform patch [all pixels active and driven at the same Digital Driving Level (DDL)] at DDL $(0,0,0)$ is shown on the screen during one hour prior to the measurements such that the temperature of the display $T_{\text {display }}$ can adapt to the ambient temperature. When a full-screen uniform patch at DDL $(0,0,0)$ is applied on the screen, there is limited heat creation, mainly coming from the electronics at the back of the display. This causes the display temperature to stabilize at approximately $1{ }^{\circ} \mathrm{C}$ above the ambient temperature in the chamber, $T_{\text {display }}=T_{\text {amb }}+1^{\circ} \mathrm{C}$.

Once the display temperature has stabilized, measurements are started. Sequentially, uniform full screen patches at different DDLs are shown on the screen. For each patch, the luminance is measured in the center of the left half of the display (the right half of the display was used for lifetime measurements and therefore no longer useful). All luminance measurements are executed using a Konica Minolta CA-210 Display Color Analyzer. The measurement head of the Konica Minolta CA-210 is placed perpendicular to the display surface. For each patch, also the power consumption of the display is measured using a Chroma 66200 Digital Power Meter. Each patch is shown on the screen and measured for six seconds and thereafter a uniform full screen patch at DDL $(0,0,0)$ is shown for 54 seconds. In this
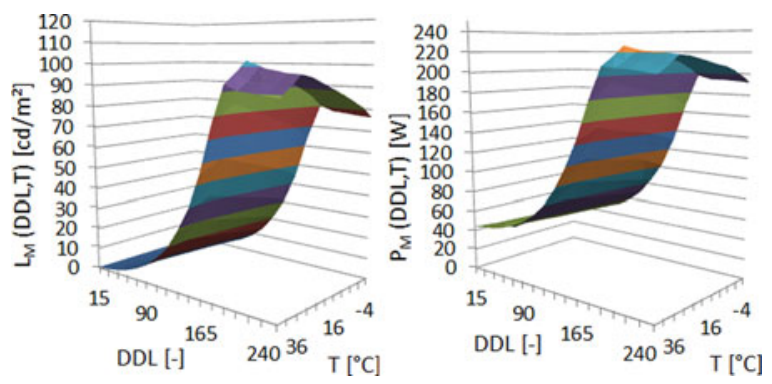

Fig. 1. Left: Luminance $L_{M}(D D L, T)$ of a 55 inch WRGB OLED display, measured in the center of the left half of the display, as a function of the $D D L$ of the applied full-screen uniform patch and the temperature $T$ of the display. Right: Power consumption $P_{M}(D D L, T)$ of a 55 inch WRGB OLED display, as a function of the $D D L$ of the applied uniform full-screen patch and the temperature $T$ of the display. The figures show the results for grayscale patches, i.e., DDLs of the form $(x, x, x)$ with $x$ ranging from 15 to 255 with step-size 15 .

way, one patch per minute is measured. In total, 52 different patches are shown on the screen. The DDLs of the patches are the following: $(252,255,215)$ (white sub-pixel only), $(x, 0,0)$ (red sub-pixel only), $(0, x, 0)$ (green sub-pixel only), $(0,0, x)$ (blue sub-pixel only) and $(x, x, x)$ (grayscale colors) with $x$ ranging from 15 to 255 with step-size 15 .

By keeping the time in which each patch is shown short (six seconds), it is guaranteed that the temperature of the OLED and TFT ( $\left.T_{\mathrm{OLED} / \mathrm{TFT}}\right)$ is kept very close to temperature of the display $\left(T_{\text {display }}\right)$ at all time. In the remainder of this section we will repeatedly use the term 'temperature of the display' or its notation $T$, thereby referring to both the temperature of the display $T_{\text {display }}$ and the temperature of the OLED and TFT $T_{\mathrm{OLED}} / \mathrm{TFT}$, with $T=T_{\text {display }}=T_{\mathrm{OLED}} / \mathrm{TFT}$.

The results of the measurements are described in Section III-A (luminance) and Section III-B (power).

\section{A. Luminance}

The results of the luminance measurements are shown in Fig. 1 (left) for grayscale colors, i.e., DDLs of the form $(x, x, x)$ with $x$ ranging from 15 to 255 with step-size 15 . Similar figures can be made for other colors. The figure shows the luminance measured in the center of the left half of the display, $L_{M}(D D L, T)$, as a function of the $D D L$ and the temperature $T$ of the display.

The results show a gamma curve linking DDL to luminance for the low DDLs and a saturation of the luminance for high DDLs. The latter is typical behavior of an OLED display when all the pixels in the display are active. Indeed, it is well-known that the luminance characteristic of an OLED display is a function of the number of active pixels. The figure also shows a non-negligible dependence of the luminance on temperature. This dependency will be investigated further in the next paragraphs.

The luminance $L_{M}(T)$ is shown as a function of temperature $T$ in Fig. 2, for DDL $(252,255,215)$ (white sub-pixel only), $(255,0,0)$ (red sub-pixel only), $(0,255,0)$ (green subpixel only), $(0,0,255)$ (blue sub-pixel only) and $(255,255$, 255) (nominal white). Fig. 3 shows the luminance $L_{M}(T)$ as a 


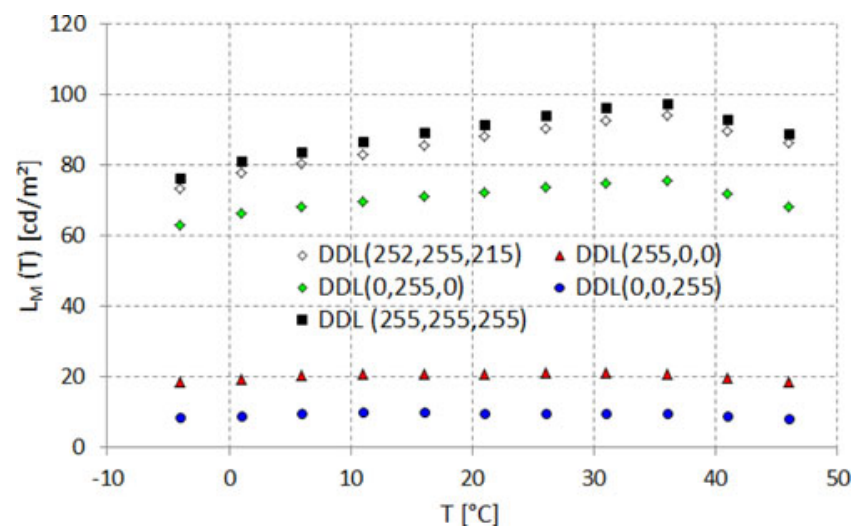

Fig. 2. Luminance $L_{M}(T)$ of a 55 inch WRGB OLED display, measured in the center of the left half of the display, as a function of temperature $T$ of the display, when uniform full-screen patches at DDL $(252,255,215)$ (white sub-pixel only), $(255,0,0)$ (red sub-pixel only), $(0,255,0)$ (green sub-pixel only) and $(0,0,255)$ (blue sub-pixel only) and $(255,255,255)$ (nominal white), are displayed.

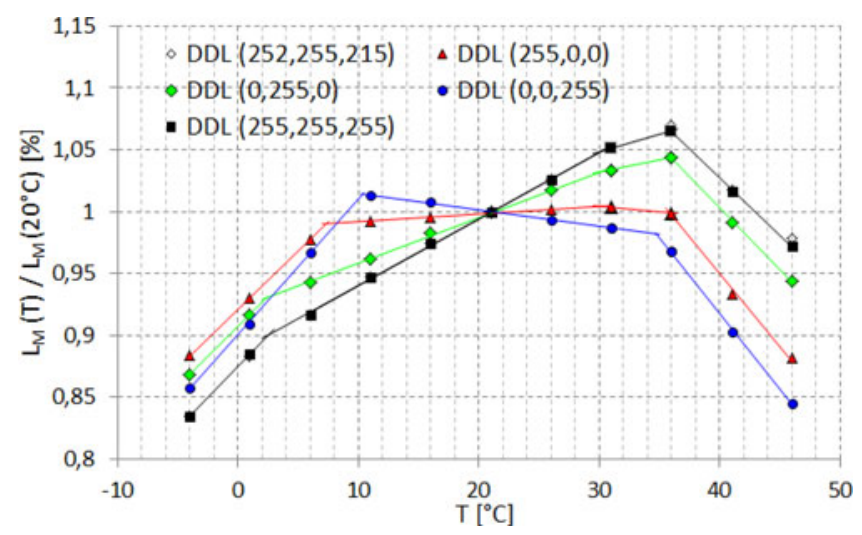

Fig. 3. Luminance $L_{M}(T)$ of a 55 inch WRGB OLED display, measured in the center of the left half of the display, relative to the luminance at 20 ${ }^{\circ} \mathrm{C} L_{M}\left(20^{\circ} \mathrm{C}\right)$, as a function of temperature $T$ of the display, when uniform full-screen patches at DDL $(252,255,215)$ (white sub-pixel only), $(255,0,0)$ (red sub-pixel only), $(0,255,0)$ (green sub-pixel only) and $(0,0,255)$ (blue sub-pixel only) and $(255,255,255)$ (nominal white) are displayed. The range of temperatures from $-5^{\circ} \mathrm{C}$ to $45^{\circ} \mathrm{C}$ can be split up in four zones. The temperature dependency within each zone can be approximated by a straight line indicating a linear relationship. The transition points between zone 1, zone 2, zone 3 and zone 4 are different for each color.

function of temperature $T$, relative to the luminance $L_{M}\left(20^{\circ} \mathrm{C}\right)$ at $20^{\circ} \mathrm{C}$. One can see that the behavior as a function of temperature varies depending on the color component. In some temperature ranges there is a monotonic increase in temperature and in other temperature ranges there is a monotonic decrease in temperature. The transition temperature at which the curves change from increasing to decreasing depends on the color.

The reason for the presence of the transition points is unclear but we assume that the OLED display houses a controller (hardware or software) that adjusts the driving of the pixels as a function of temperature. There are several reasons for implementing such a controller. Firstly, it can compensate for the temperature dependency of the display's luminance. Indeed, by scaling back the voltage applied to the gate of the TFT as temperature increases, one can compensate for the increase in luminance

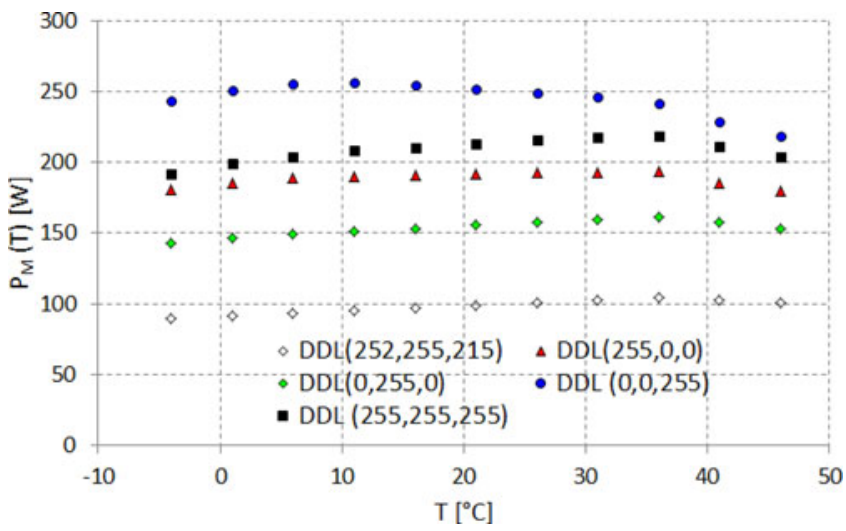

Fig. 4. Power consumption $P_{M}(T)$ of a 55 inch WRGB OLED display, as a function of temperature $T$ of the display, when uniform full-screen patches at DDL $(252,255,215)$ (white sub-pixel only), $(255,0,0)$ (red sub-pixel only), (0, $255,0)$ (green sub-pixel only), $(0,0,255)$ (blue sub-pixel only) and $(255,255$, 255) (nominal white), are displayed.

resulting from the temperature increase. Secondly, such a controller can be useful for reducing the aging of the OLED display. Indeed, it is well-known that OLED aging is accelerated at elevated temperatures. By scaling back the driving of the pixels at high temperatures, one can reduce this effect. Since the OLED display is very likely housing a controller, we could assume that only the temperature range left of the first transition point is representing the actual temperature dependence of the OLEDs, and the influence of temperature on the luminance would be much stronger if no controller were present.

\section{B. Power}

The results of the power measurements are shown in Fig. 1 (right) for grayscale colors, i.e., DDLs of the form $(x, x, x)$ with $x$ ranging from 15 to 255 with step-size 15 . Similar figures can be made for other colors. The figure shows the measured power consumption of the display $P_{M}(D D L, T)$, as a function of the $D D L$ and the temperature $T$ of the display.

At DDL $(0,0,0)$, one notices a power offset of approximately $42 \mathrm{~W}$. This is the power consumption of the electronic boards at the back of the display [4]. Further, one notices that the shape of the power consumption curve is very similar to the luminance curve, with a gamma curve linking power consumption to DDL for the low DDLs, a saturation of the power consumption for high DDLs and a non-negligible dependence of the power consumption on temperature. In a similar way as for luminance, we investigate this temperature dependency in the next paragraphs.

The power consumption $P_{M}(T)$ is shown as a function of temperature $T$ in Fig. 4, for DDL $(252,255,215)$ (white subpixel only), $(255,0,0)$ (red sub-pixel only), $(0,255,0)$ (green sub-pixel only), (0, 0, 255) (blue sub-pixel only) and $(255,255$, 255) (nominal white). Fig. 5 shows the power consumption $P_{M}(T)$ as a function of temperature $T$, relative to the power consumption $P_{M}\left(20^{\circ} \mathrm{C}\right)$ at $20^{\circ} \mathrm{C}$. Similarly as for the luminance, one can see that the behavior as a function of temperature depends on the color component, with for each color, temperature ranges in which there is a monotonic increase in power 


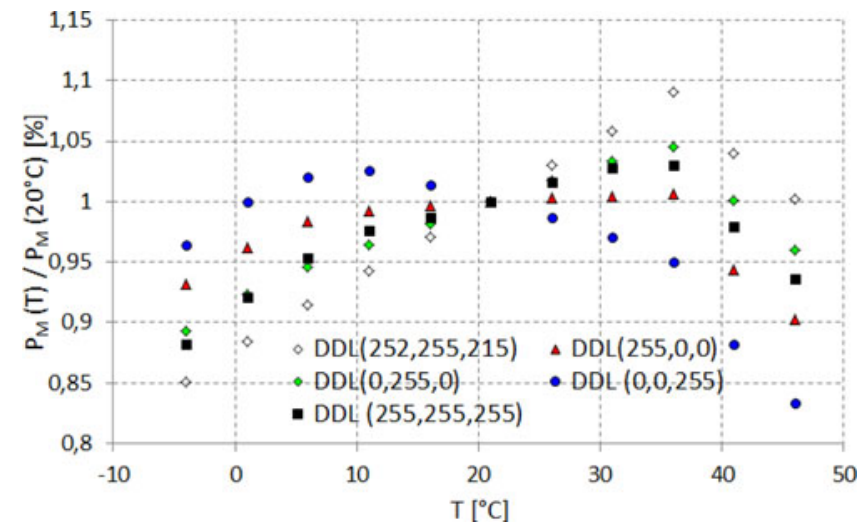

Fig. 5. Power consumption $P_{M}(T)$ of a 55 inch WRGB OLED display, relative to the power consumption at $20^{\circ} \mathrm{C} P_{M}\left(20^{\circ} \mathrm{C}\right)$, as a function of the temperature $T$ of the display, when uniform full-screen patches at DDL (252, $255,215)$ (white sub-pixel only), $(255,0,0)$ (red sub-pixel only), $(0,255,0)$ (green sub-pixel only), $(0,0,255)$ (blue sub-pixel only) and $(255,255,255)$ (nominal white), are displayed.

consumption and temperature ranges in which there is a monotonic decrease. Also the transition points at which the curves change from increasing to decreasing is different depending on the color.

\section{Luminance at Equal Power Consumption for Different Sub-Pixels}

In the previous sections it was described that the temperature dependency of the luminance and power consumption of the display depends on the color. In this section, this difference will be investigated further.

In a WRGB OLED display, each of the sub-pixels (white, red, green and blue) is based on a white OLED [4]. The white light emitted by the OLED is filtered to convert it into colored light. For each color primary, the display is calibrated to emit a specific amount of light, according to the preferences of the manufacturer. Both the amount of light that needs to be emitted and the transmission of the filter determine the power at which the white OLED in each of the sub-pixels needs to be driven. This is different for each color.

This means that the five curves in Figs. 2, 3, 4 and 5 are showing the behavior of the white OLED, but at different intensity levels for each color. The DDLs that lead to equal driving of the white OLEDs inside the different sub-pixels are found as those DDLs for which the total power consumption of the display is the same. As we only have measurements for DDL $(252,255,215)$ and DDLs of the form $(x, y, z)$, with $x, y$ and $z$ ranging from 15 to 255 with step 15 , we choose for each color a DDL such that the power consumptions are as closely together as possible. The result is shown in Fig. 6 for luminance and Fig. 7 for power consumption. The figures show the relative change in luminance and power consumption as a function of temperature with respect to the luminance and power consumption at $20^{\circ} \mathrm{C}$ for DDL $(252,255,215)$ (white sub-pixel only), DDL $(150,0,0)$ (red sub-pixel only), DDL $(0,150,0)$ (green sub-pixel only) and DDL $(0,0,135)$ (blue sub-pixel

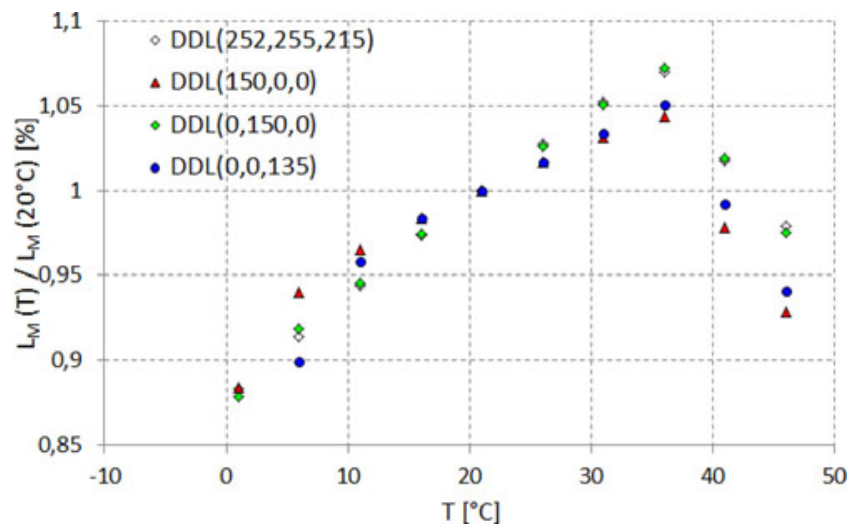

Fig. 6. Luminance $L_{M}(T)$ of a 55 inch WRGB OLED display, measured in the center of the left half of the display, relative to the luminance at $20^{\circ} \mathrm{C}$ $L_{M}\left(20^{\circ} \mathrm{C}\right)$, as a function of temperature $T$ of the display, when uniform fullscreen patches at DDL $(150,0,0)$ (red sub-pixel only), DDL $(0,150,0)$ (green sub-pixel only), DDL (0, 0, 135) (blue sub-pixel only) and DDL (252, 255, 215) (white sub-pixel only) are displayed. The driving levels for the different colors were chosen such that the power consumption when showing the uniform full-screen patch is similar for the different color components.

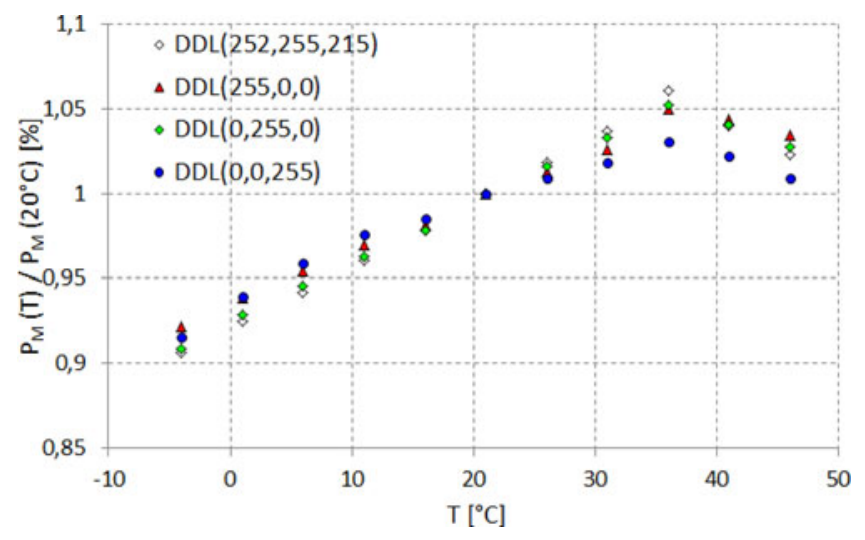

Fig. 7. Power consumption $P_{M}(T)$ of a 55 inch WRGB OLED display, relative to the power consumption at $20^{\circ} \mathrm{C} P_{M}\left(20^{\circ} \mathrm{C}\right)$, as a function of temperature $T$ of the display, when uniform full-screen patches at DDL $(150,0,0)$ (red sub-pixel only), DDL $(0,150,0)$ (green sub-pixel only), DDL $(0,0,135)$ (blue sub-pixel only) and DDL $(252,255,215)$ (white sub-pixel only) are displayed. The driving levels for the different colors were chosen such that the power consumption when showing the uniform full-screen patch is similar for the different color components.

only). The power consumption for these driving levels is respectively $99.0 \mathrm{~W}, 100.2 \mathrm{~W}, 89.9 \mathrm{~W}$ and $96.7 \mathrm{~W}$. It can be seen that the shape of the curves representing the relation between temperature and luminance for the different colors are indeed similar.

\section{TFT Characteristic}

From the measurements of the power consumption $P_{M, i}(D D L, T)$ presented in Section III-B, with $i=1$.. 4 representing the measurements for red, green, blue and white respectively, we can estimate the current density $J_{M, i}(D D L, T)$ delivered by the TFT of sub-pixel $i$ to the OLED in sub-pixel $i$, with $i=1 . .4$ representing the red, green, blue and white 


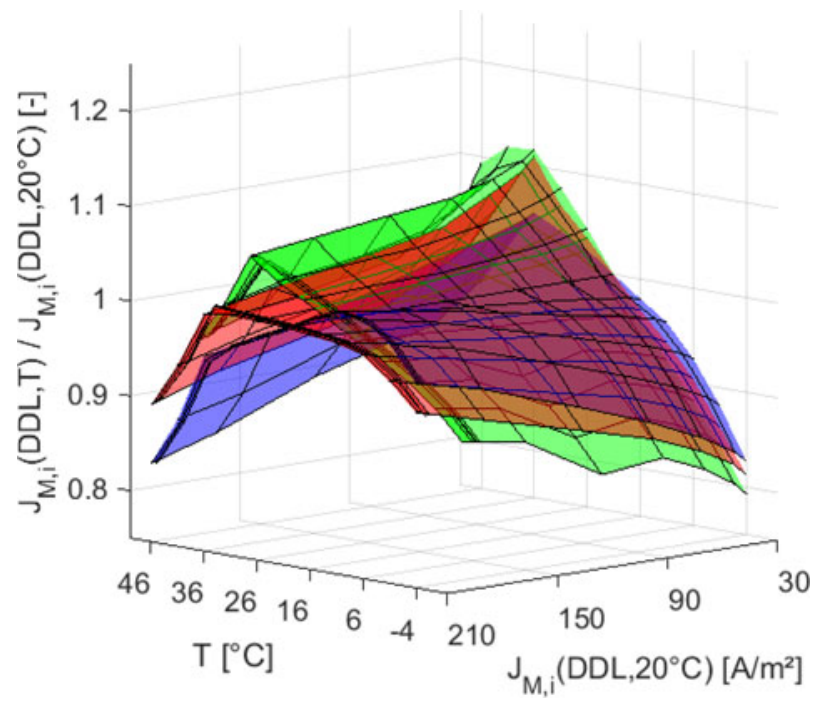

Fig. 8. Characteristic of the TFTs inside the OLED display. The figure shows the current density $J_{M, i}(D D L, T)$ delivered by the TFT to the OLED, in function of the $D D L$ of the applied full-screen uniform patch and the temperature $T$ of the display, and relative to the current density $J_{M, i}\left(D D L, 20^{\circ} \mathrm{C}\right)$ delivered by the TFT to the OLED for the same $D D L$ at $20^{\circ} \mathrm{C}$.

sub-pixel respectively, using the following formula:

$$
P_{M, i}(D D L, T)=\sum_{i} N V_{\mathrm{DD}} J_{M, i}(D D L, T) A F_{i}+P_{\mathrm{offset}}
$$

with $N[-]$ the number of pixels in the display, $V_{\mathrm{DD}}[V]$ the column voltage (i.e., the total voltage across the OLED, the TFT and the power line), $A\left[\mathrm{~m}^{2}\right]$ the area of a pixel, $F_{i}[-]$ the fill factor of sub-pixel $i$ and $P_{\text {offset }}[W]$ the power consumed by the display when the screen is completely black.

Fig. 8 shows the current density $J_{M, i}(D D L, T)$ delivered by the TFT to the OLED, relative to the current density delivered by the TFT to the OLED at $20^{\circ} \mathrm{C} J_{M, i}\left(D D L, 20^{\circ} \mathrm{C}\right)$, as a function of the temperature of the TFT $T$ and the current density $J_{M, i}\left(D D L, 20^{\circ} \mathrm{C}\right)$. In an AMOLED display we expect that the current is determined by the gate voltage of the TFT and the temperature dependency of the TFT and of the OLED.

It can be seen from the figure that the behavior of the different color components is very similar, with moderate relative changes of the power consumption as a function of temperature for the high driving levels, and increasingly larger relative changes of the power consumption as a function of temperature as the driving level drops. This result indicates that the TFT contributes significantly to the temperature dependency of the OLED luminance.

\section{E. OLED Efficiency}

From the calculated current density $J_{M, i}(D D L, T)$ and the measurements of the luminance $L_{M, i}(D D L, T)$ presented in Section III-A, with $i=1 . .3$ representing the measurements for red, green and blue respectively, we can calculate the efficacy of the white OLED $\eta_{M, i}\left(J_{M, i}, T\right)[W /(s r . A)]$, using the following

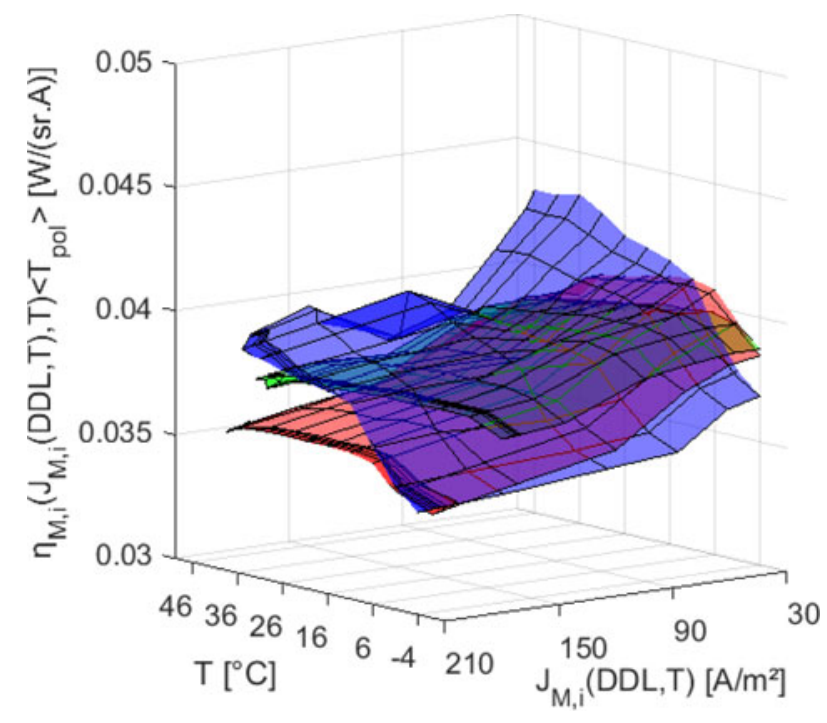

Fig. 9. Efficiency $\eta_{M, i}\left(J_{M, i}, T\right)$ of the white OLEDs inside the OLED display as a function of the current density $J_{M, i}$ flowing through sub-pixel $i$ and the temperature $T$.

formula:

$$
\eta_{M, i}\left(J_{M, i}, T\right)\left\langle T_{\mathrm{pol}}\right\rangle=\frac{L_{M, i}(D D L, T)}{J_{M, i}(D D L, T) F_{i}\left\langle T_{i}\right\rangle\left\langle\bar{y}_{i}\right\rangle}
$$

with $\left\langle T_{i}\right\rangle[-]$ the transmission of the filter in front of subpixel $i,\left\langle T_{\mathrm{pol}}\right\rangle[-]$ the transmission of the polarizer and $\left\langle\bar{y}_{i}\right\rangle$ $[\mathrm{lm} / W]$ the eye sensitivity. $\left\langle T_{i}\right\rangle,\left\langle T_{\mathrm{pol}}\right\rangle$ and $\left\langle\bar{y}_{i}\right\rangle$ are averages of the wavelength dependent characteristics weighted with the spectrum of the white light [4].

The resulting efficiency $\eta_{M, i}\left(J_{M, i}, T\right)$ is shown in Fig. 9. The figure shows a decrease in efficiency for increasing current density and a limited influence of temperature on the efficiency. The influence of temperature is stronger for those driving levels for which there is intervention of the controller (mainly blue).

\section{DYNAMIC TEMPERATURE VARIATION}

In this section, the influence of temperature on the luminance of an OLED display is investigated in a dynamic context.

\section{A. Full Screen Transient}

To investigate the influence of temperature on the luminance of an OLED display in a dynamic context, four experiments are set up in which uniform full-screen patches, respectively at DDL $(255,255,255)$ (nominal white), $(255,0,0)$ (red subpixel only), $(0,255,0)$ (green sub-pixel only) and $(0,0,255)$ (blue sub-pixel only), are displayed on the screen for two hours continuously. Throughout this period, the temperature of the display and the luminance of the display are measured in the center of the left half of the display. The temperature is measured using a FLIR A320 IR camera with a resolution of $320 \times 240$ pixels. The luminance is measured using a different device than in Section III. This time, the luminance is measured using a CCD camera with a resolution of $2184 \times 1472$ because it allows measuring the luminance across the entire display area. The 


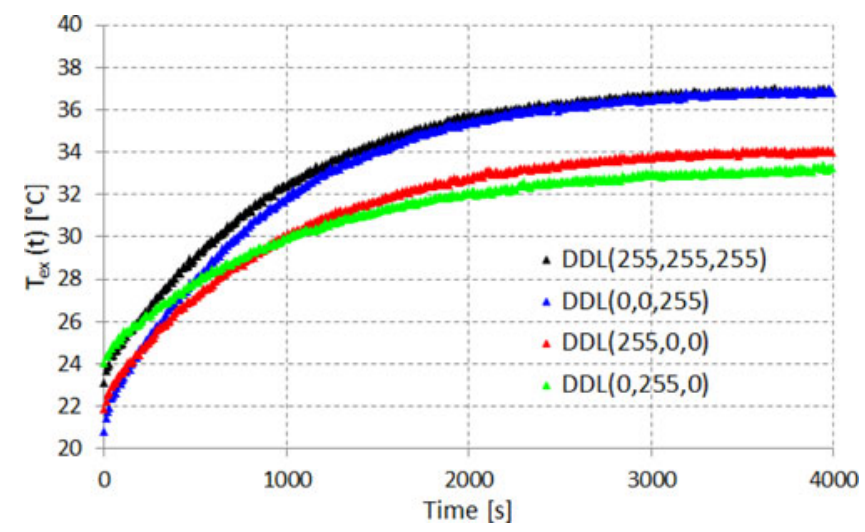

Fig. 10. Measured temperature $T_{\mathrm{ex}}(t)$ in the center of the left half of the display when a full-screen uniform patch at $\operatorname{DDL}(255,255,255),(255,0,0)$, $(0,255,0)$ and $(0,0,255)$ is shown on the screen for two hours continuously.

luminance at one specific location is then calculated by taking an average across that specific location in the image. The same approach is used for calculating the temperature at one specific location based on the IR camera measurements. Luminance as measured by the Konica Minolta CA-210 (Section III) can be converted into luminance as measured by the CCD camera and vica versa. Finally, also the power consumption of the display is measured continuously by means of a Chroma 66200 Digital Power Meter. The results of the temperature, luminance and power measurements are shown respectively in Figs. 10, 11 and 12 .

Fig. 10 shows a strong increase in measured temperature $T_{\text {ex }}(t)$ of the display over time, resulting from the power losses in the display. After approximately 1 hour, a steady-state temperature is reached in the experiments, with blue, white, red and green in decreasing order of maximum temperature. The experiments were executed in a room without ambient temperature control. This causes slight offsets in the temperature of the display between different measurements. Fig. 11 shows the measured change in luminance $L_{\mathrm{ex}}(T)$ throughout the measurement period, for each of the colors. For white and green, the luminance is monotonically increasing. For blue and red, the luminance is not monotonically increasing, with an initial rise in luminance followed by a decrease in luminance. Fig. 12 shows the change in measured power $P_{\mathrm{ex}}(T)$ throughout the measurement period. The changes in power are similar to the changes in luminance.

\section{B. Interpolation of $L_{M}(T)$}

The link between temperature and luminance presented in Section III resulted from measurements that were executed in a controlled temperature environment and in such a way that the temperature of the display, including the OLED and TFT, was kept constant. Therefore these measurements link the luminance of the display to the actual temperature of the OLED and TFT for a static temperature. In this section we will investigate whether the same link can be used in a dynamic context, i.e., whether it allows estimating the change in luminance resulting from a transient temperature.

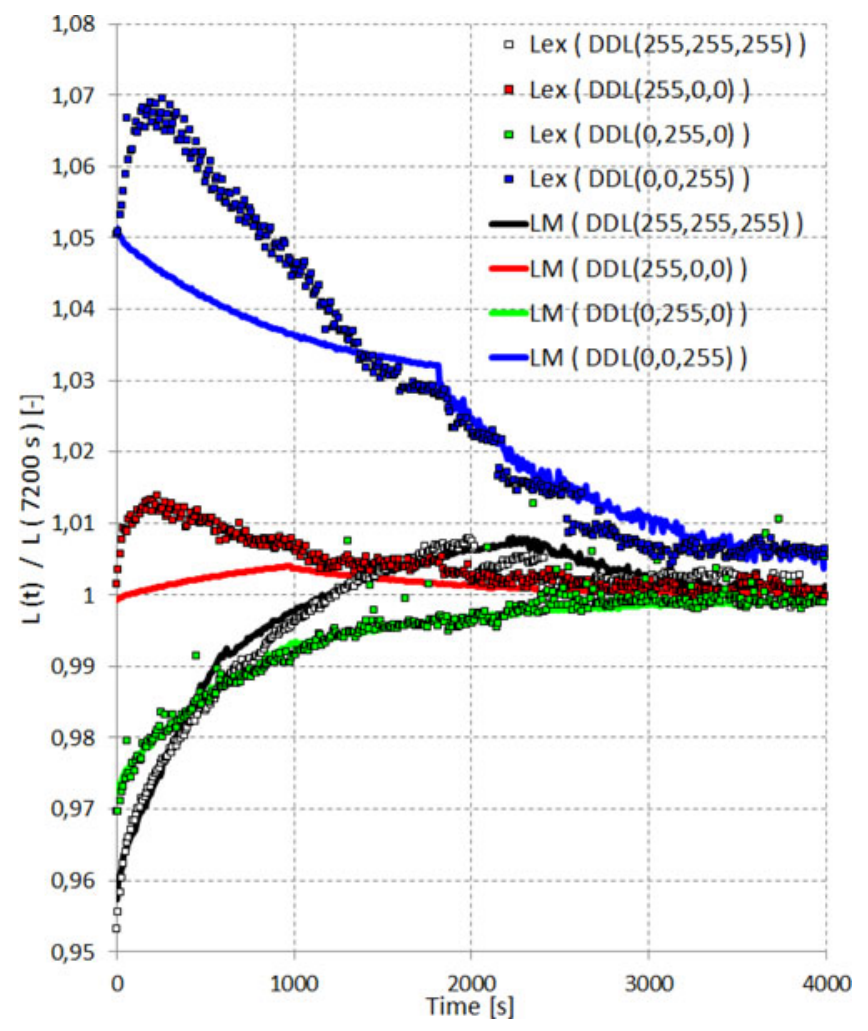

Fig. 11. Measured luminance $L_{\text {ex }}(t)$ in the center of the left half of the display when a full-screen uniform patch at $\operatorname{DDL}(255,255,255),(255,0,0),(0,255,0)$ and $(0,0,255)$ is shown on the screen for two hours continuously (dots), relative to the luminance $L_{\mathrm{ex}}(7200 \mathrm{~s})$ of the display at the end of the measurements Luminance $L_{M}\left(D D L, T_{\mathrm{ex}}(t), t\right)$, estimated from the measured temperature $T_{\mathrm{ex}}(t)$ (lines).

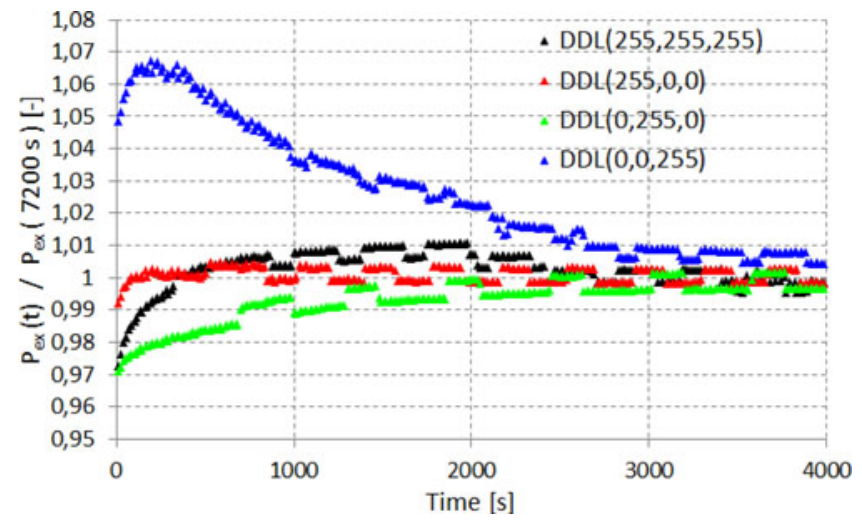

Fig. 12. Measured power consumption $P_{\mathrm{ex}}(t)$ of the display when a fullscreen uniform patch at DDL $(255,255,255),(255,0,0),(0,255,0)$ and $(0,0$, $255)$ is shown on the screen for two hours continuously, relative to the power consumption $P_{\mathrm{ex}}(7200 \mathrm{~s})$ of the display at the end of the measurements.

Taking a closer look at Fig. 3, we can distinguish four different zones in the temperature dependency of the luminance of the display for each color. Within each zone, the temperature dependency of the luminance can be approximated by a straight line. The transition points between the zones are different for each color. The equations for the straight lines as well as the transition points between the zones are listed in Table I. The information in Table I allows calculating the luminance of 
TABLE I

EQUATIONS OF THE STRAIGHT LINES APPROXIMATING THE RELATIONSHIP BETWEEN LUMINANCE AND TEMPERATURE FOR WHITE, RED, GREEN AND BLUE, UNDER STATIC TEMPERATURE CONDITIONS

\begin{tabular}{ccccc}
\hline \hline & Zone 1 & Zone 2 & Zone 3 & Zone 4 \\
\hline White & $0.0100^{*} \mathrm{~T}$ & $0.0054^{*} \mathrm{~T}$ & $0.0028^{*} \mathrm{~T}$ & $-0.0093^{*} \mathrm{~T}$ \\
& +0.8748 & +0.8867 & +0.9634 & +1.3999 \\
& $<2.5^{\circ} \mathrm{C}$ & {$\left[2.5^{\circ} \mathrm{C}, 30^{\circ} \mathrm{C}\right]$} & {$\left[30^{\circ} \mathrm{C}, 36^{\circ} \mathrm{C}\right]$} & $>36^{\circ} \mathrm{C}$ \\
Red & $0.0094^{*} \mathrm{~T}$ & $0.0006^{*} \mathrm{~T}$ & $-0.0009^{*} \mathrm{~T}$ & $-0.0118^{*} \mathrm{~T}$ \\
& +0.921 & +0.9860 & +1.0307 & +1.4200 \\
& $<7.5^{\circ} \mathrm{C}$ & {$\left[7.5^{\circ} \mathrm{C}, 30^{\circ} \mathrm{C}\right]$} & {$\left[30^{\circ} \mathrm{C}, 36^{\circ} \mathrm{C}\right]$} & $>36^{\circ} \mathrm{C}$ \\
Green & $0.0097^{*} \mathrm{~T}$ & $0.0037^{*} \mathrm{~T}$ & $0.002^{*} \mathrm{~T}$ & $-0.01^{*} \mathrm{~T}$ \\
& +0.9069 & +0.9220 & +0.9724 & +1.4025 \\
& $<2.5^{\circ} \mathrm{C}$ & {$\left[2.5^{\circ} \mathrm{C}, 30^{\circ} \mathrm{C}\right]$} & {$\left[30^{\circ} \mathrm{C}, 36^{\circ} \mathrm{C}\right]$} & $>36^{\circ} \mathrm{C}$ \\
Blue & $0.0109^{*} \mathrm{~T}$ & $-0.0013^{*} \mathrm{~T}$ & $-0.0013^{*} \mathrm{~T}$ & $-0.0123^{*} \mathrm{~T}$ \\
& +0.9015 & +1.0284 & +1.0284 & +1.4111 \\
& $<9.5^{\circ} \mathrm{C}$ & {$\left[9.5^{\circ} \mathrm{C}, 30^{\circ} \mathrm{C}\right]$} & {$\left[30^{\circ} \mathrm{C}, 35^{\circ} \mathrm{C}\right]$} & $>35^{\circ} \mathrm{C}$ \\
\hline \hline
\end{tabular}

Also the Temperatures at Which the Transitions Between the Zones Take Place Are Indicated. The Lines Are Shown in Fig. 3.

the display for a full-screen uniform patch at DDL $(255,255$, 255) (nominal white), DDL $(255,0,0)$ (red sub-pixel only), DDL $(0,255,0)$ (green sub-pixel only) and DDL $(0,0,255)$ (blue sub-pixel only), for a given temperature. Such tables can also be constructed for other driving levels or other patch sizes, when the measurements are available.

The results in Table I are used to estimate the change in luminance $L_{M}\left(D D L, T_{\mathrm{ex}}(t)\right)$ resulting from the measured change in temperature $T_{\mathrm{ex}}(t)$, shown in Fig. 10. For each temperature point, the corresponding luminance is calculated using the equations in Table I. The luminance estimations $L_{M}\left(D D L, T_{\mathrm{ex}}(t)\right)$ are shown together with the luminance measurements $L_{\mathrm{ex}}(t)$ in Fig. 11 for white, red, green and blue.

It can be seen that there is good correspondence between the measurements and the estimations in the initial state (the first measurement point, before any temperature rise) and final state of the measurements (when a steady-state temperature has been reached). Mainly for red and blue there is a deviation between the estimated and measured luminance in parts of the curve. There are two reasons for this deviation. One reason is that the temperature is measured using an IR camera pointed at the front of the display. As the front glass of the display is not transparent to thermal radiation, the IR camera is measuring the temperature of the front glass rather than the temperature of the OLED and TFT. As the heat source causing the rise in temperature is in these experiments the OLED and TFT, it is expected that the temperature of the front glass will rise more slowly than the temperature of the OLED and TFT, taking into account the thermal barriers between the OLED/TFT and the front glass and the heat capacity of the display. Another reason for the deviation between the estimated and the measured luminance is found by looking back at Fig. 3. One can see that for red and blue, the increase in luminance as a function of temperature is much less pronounced than for the other colors, indicating the intervention of a controller. Indeed, most likely, the voltage applied to the gate of the TFTs is for these colors scaled back as a function of temperature, even for moderate temperatures below $35^{\circ} \mathrm{C}$. This is the case because blue and red are the most sensitive to OLED aging. The measurements in Fig. 11 show that the intervention of the controller is not instantaneous with a short period of increase before the luminance starts dropping as a result of the temperature increase. A possible reason for this could be that the temperature sensing, which is provided as input to the controller, is located at a certain distance from the heat source.

\section{Small AREa Transient Luminance}

It has been observed that OLED displays suffer in some circumstances from temporary image sticking after the switch to another image. For example, a pattern displayed on the screen for a long time can under circumstances remain visible after switching to a uniform image. This effect is most clearly visible for large changes in driving level (e.g., from DDL 255 to DDL 60), when the driving level after the switch is low, when the image before the switch contains sharp edges and when the first image was applied for a sufficiently long time. It should be emphasized that this effect is not related to permanent image sticking or burn-in in an OLED display which is the result of OLED aging and causes a permanent decrease in the light output of an OLED as a function of its operating time.

In this section we investigate this effect and measure luminance and temperature during the transition between two images. To that extent, three experiments are set up. In experiment 1, a zone on the screen is switched from DDL 0 to DDL 60 (t $>7200 \mathrm{~s})$. In experiment 2 , a zone on the screen is switched from DDL 255 to DDL 60. In experiment 3, a zone on the screen is switched from DDL 0 to DDL 60, but the pixels surrounding the zone are driven at DDL 255 before the switch, causing strong heating of the zone. The patches that were applied on the screen during each of the experiments, before and after the switch, are shown in Fig. 13. In the same way as in Section IV, a FLIR A320 IR camera is used for temperature measurements and a CCD camera is used for luminance measurements.

We are interested in what happens after the switch to DDL 60. The measurements of the temperature $T_{\mathrm{ex}}(T)$ are shown in Fig. 14. The measurements of the luminance $L_{\text {ex }}(T)$ are shown in Fig. 15. When comparing experiment 1 and 2 it can be seen that the patch that was applied prior to the switch has an influence on the luminance after the switch. We see that this effect is even stronger in experiment 3 . The measurements of experiment 3 show that the patch that is applied before the switch is having a large impact on the luminance after the switch with a difference of approximately $30 \%$ between the luminance right after the switch and when a steady state has been reached. It is found that the luminance right after the switch corresponds to a DDL of 67 instead of the desired DDL of 60 and that this luminance deviation corresponds to 16 JNDs in the DICOM Grayscale Standard Display Function (GSDF) [22]. Further, as the luminance deviation is local, it causes luminance non-uniformity across the screen. For medical displays, the difference in luminance, measured at five different locations across the screen (center and four corners), should not exceed $30 \%$ [23]. In practice, a more stringent requirement is used for medical displays whereby the 

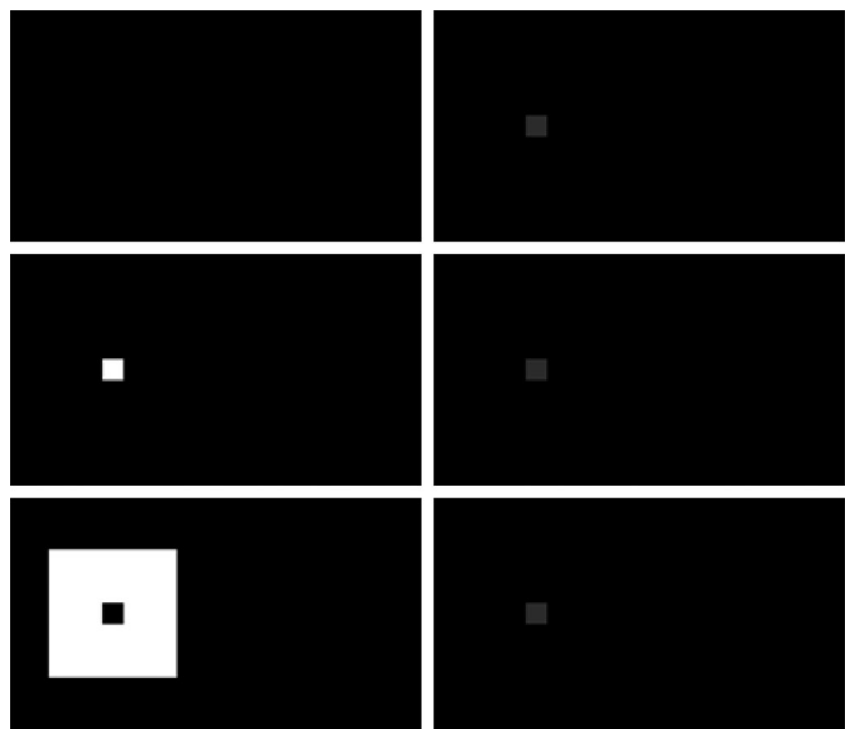

Fig. 13. Patches of DDL levels applied on the screen, before (DDL 0 or DDL 255) (left) and after (DDL 0 or DDL 60) (right) the switch, for experiment 1 (top), experiment 2 (middle) and experiment 3 (bottom).

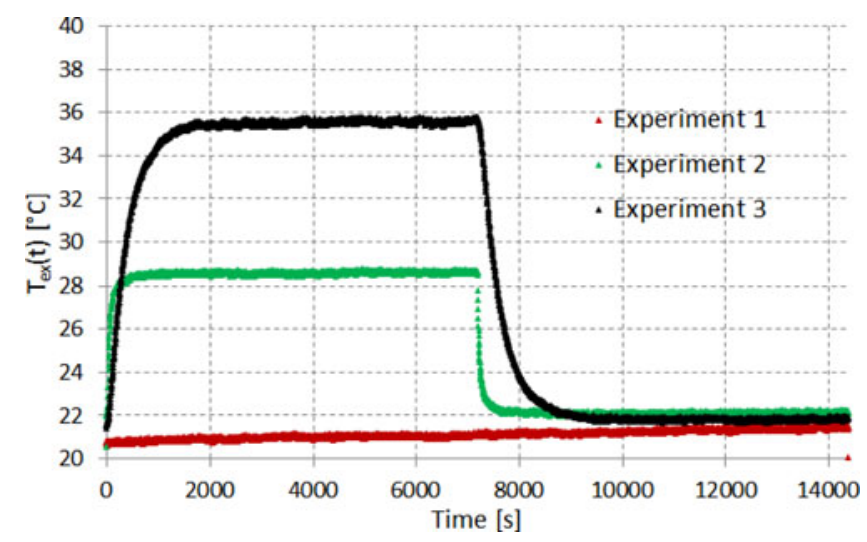

Fig. 14. Measured temperature $T_{\mathrm{ex}}(t)$ of the display for experiments 1, experiment 2 and experiment 3 .

luminance difference should not exceed $7 \%$. The aformentioned figures suggest that the luminance deviation of $30 \%$ arising in this experiment is significant and not acceptable for professional applications, such as medical imaging.

Now we estimate the luminance change after the switch using the same approach as used in Section IV-B. In Fig. 16, the measured luminance $L_{M}(T)$ as a function of temperature $T$ is shown for a small square at DDL 60 and DDL 255, in a static context. We use this relation to estimate the luminance variation $L_{M}\left(D D L, T_{\mathrm{ex}}(t)\right)$ based on the temperature variation $T_{\mathrm{ex}}(t)$ in Fig. 14. The results are shown, together with the luminance measurements, in Fig. 15. The results show that the estimated luminance matches well with the measured luminance for each of the experiments. Only a minor part of the change in luminance cannot be attributed to the variation in temperature. Those effects could be the result of control mechanisms in the display that adjust the current over time.

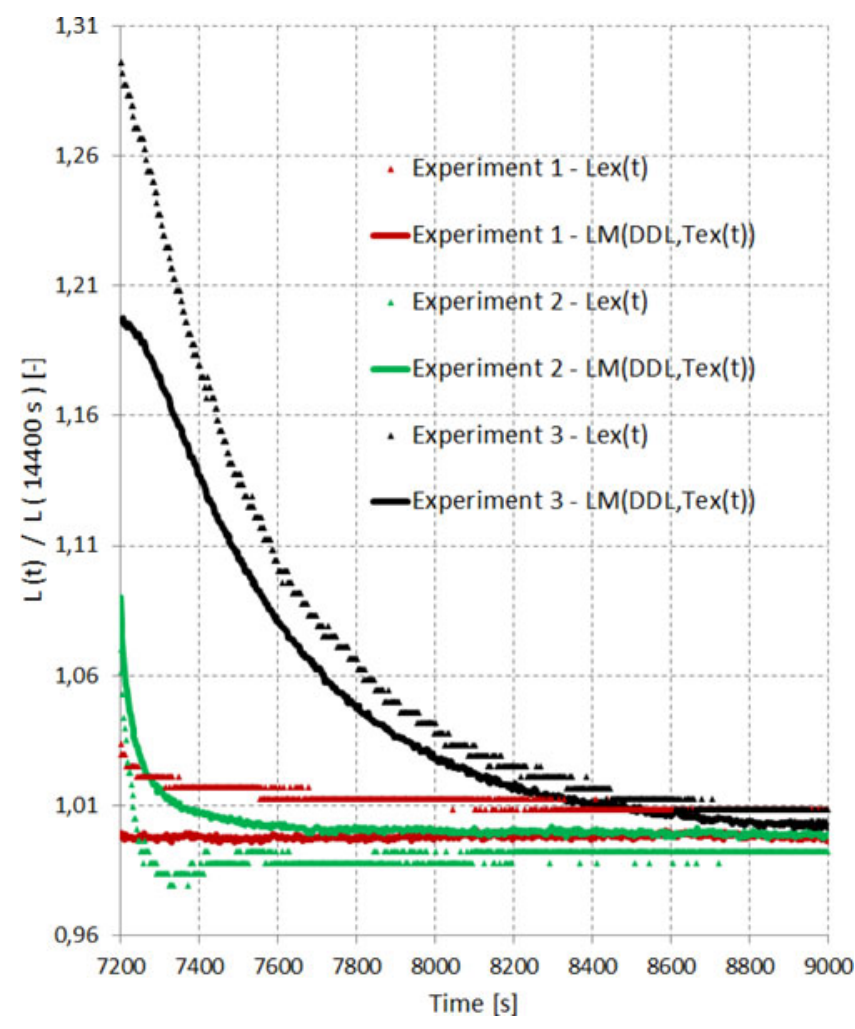

Fig. 15. Measured luminance $L_{\mathrm{ex}}(t)$ of the display for experiment 1, experiment 2 and experiment 3 , relative to the luminance $L_{\mathrm{ex}}(14400 \mathrm{~s})$ two hours after the switch (dots). Luminance $L_{M}\left(D D L, T_{\mathrm{ex}}(t)\right)$, estimated from the measured temperature $T_{\mathrm{ex}}(t)$ (lines).

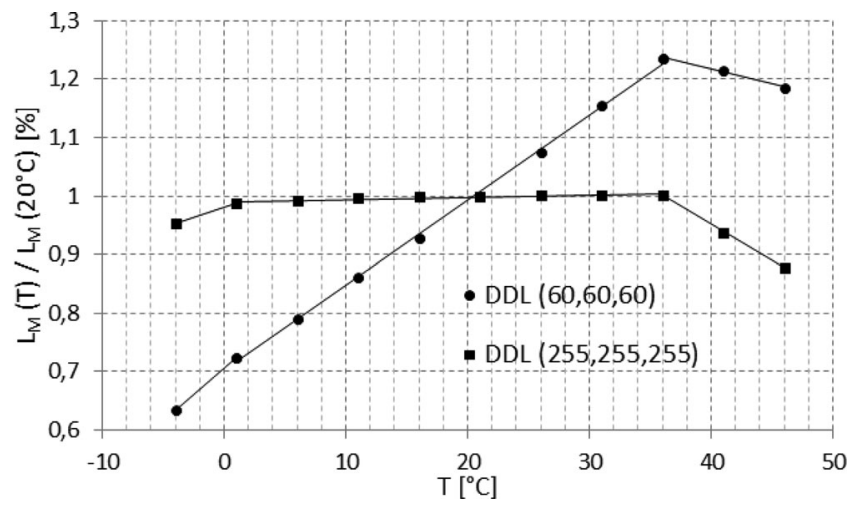

Fig. 16. Luminance $L_{M}(T)$ as a function of temperature $T$, relative to the luminance $L_{M}\left(20^{\circ} \mathrm{C}\right)$ at $20^{\circ} \mathrm{C}$, for a small square at DDL $(60,60,60)$ and $(255,255,255)$.

The measurements described in this section and the match with the simulations (based on the link between temperature and luminance from Section III) explain why image sticking is observed under some circumstances. Indeed, a pattern consisting of a bright zone (high DDL) surrounded by a dim zone (low DDL) will cause local heating. When afterwards a uniform image is applied, the local hot spot will result in a local peak in luminance, fading away slowly as the spot cools down. An observer will experience this effect as image sticking since the zone that previously was at high DDL will be slightly visible after switching. 


\section{CONCLUSION}

In this paper, we investigated the temperature dependency of the luminance of an OLED display. Measurements on a 55" WRGB active-matrix OLED display with a resolution of $1920 \times 1080$ and an oxide-TFT backplane have shown that the luminance is influenced by temperature with strong relative changes as a function of temperature at low driving levels and more moderate changes as a function of temperature at higher driving levels. It was shown that there is a strong similarity in behavior between the different sub-pixels, which is expected as each of the sub-pixels is using the same white OLED. From the measurement results, we could calculate the current density and the efficiency of the OLED and show that both are a function of temperature.

The influence of temperature on the luminance of an OLED display was investigated in a dynamic context. It was shown that temperature changes have an important impact on the dynamic luminance of the display and that the link between temperature and luminance that was found from measurements under static temperature conditions can be used to estimate the change in luminance based on the transient temperature.

The impact of the temperature dependency on the display's luminance was shown through a number of scenarios in which a switch between patches leads to a degradation of the display's picture quality as a result of temperature changes. Indeed, in some cases, the temperature dependency leads to image sticking or retention as the temperature created when applying a certain image causes deviations of the luminance after the switch of up to 30 percent. This deviation is significant and not acceptable for medical applications. These examples show that it is essential to take into account the thermal behavior when working towards a high performing OLED display.

\section{REFERENCES}

[1] C. W. Tang and S. A. Van Slyke, "Organic electroluminescent diodes," Appl. Phys. Lett., vol. 51, no. 12, pp. 913-915, Sep. 1987.

[2] N. Odlum et al., "Preliminary display comparison for dental diagnostic applications," Proc. SPIE, vol. 8318, 2012, Art. no. 83181S.

[3] T. Kimpe and C. Marchessoux, "Important differences between medical displays and normal desktop displays and underlying reasons," Amer. Display Eng. Appl. Conf., 2006.

[4] F. Chesterman et al., "Power consumption and temperature distribution in WRGB active-matrix OLED displays," J. Display Technol., vol. PP, no. 99, pp. 1-10, Dec. 2015.

[5] F. Chesterman et al., "Temperature distribution in WRGB activematrix OLED displays," SID Symp. Dig. Tech. Papers, vol. 47, no. 1, pp. 1764-1767, 2016.

[6] J. C. Sturm, W. Wilson, and M. Iodice, "Thermal effects and scaling in organic light-emitting flat-Panel displays," IEEE J. Sel. Topics Quantum Electron., vol. 4, no. 1, pp. 75-82, Jan./Feb. 1998.

[7] M.-H. M. Lu et al., "Power consumption and temperature increase in large area active-matrix OLED displays," J. Display Technol., vol. 4, no. 1, pp. 47-53, Mar. 2008.

[8] S. Chung et al., "Substrate thermal conductivity effect on heat dissipation and lifetime improvement of organic light-emitting diodes.," Appl. Phys. Lett., vol. 94, no. 25, pp. 253302, Jun. 2009.

[9] R. S. Cok et al., "Heat distribution in AMOLEDs," J. Soc. Inf. Display, vol. 13, no. 10, pp. 849-855, Oct. 2005.

[10] K. J. Bergemann, R. Krasny, and S. R. Forrest, "Thermal properties of organic light-emitting diodes," Organic Electron., vol. 13, no. 9, pp. $1565-1568$, Sep. 2012

[11] A. Sure et al., "Thermal modeling of organic light-emitting diode display panels," J. Display Technol., vol. 11, no. 12, pp. 1048-1055, Dec. 2015.

[12] S. Berleb et al., "Temperature dependent device characteristics of organic light-emitting devices," Synthetic Metals, vol. 111, pp. 341-344, Jun. 2000.

[13] C. Gärditz et al., "Impact of Joule heating on the brightness homogeneity of organic light emitting devices," Appl. Phys. Lett., vol. 90, no. 10, Mar. 2007, Art. no. 103506.

[14] H. Mu et al., "Temperature dependence of electron mobility, electroluminescence and photoluminescence of Alq(3) in OLED," J. Phys. D: Appl. Phys., vol. 41, no. 23, Dec. 2008, Art. no. 235109.

[15] I. R. de Moraes et al., "Impact of temperature on the efficiency of organic light emitting diodes," Organic Electron., vol. 26, pp. 158-163, Nov. 2015.

[16] Z. Shen et al., "Temperature dependence of current transport and electroluminescence in vacuum deposited organic light emitting devices," Japanese J. Appl. Phys. Part 2: Lett., vol. 35, no. 3B, pp. L401-L404, Mar. 1996.

[17] G. Y. Zhong et al., "Temperature-dependent photoluminescence of organic light-emitting materials: Types and characteristics of exciton involved in the emitting process," Chemical Phys. Lett., vol. 420, no. 4-6, pp. 347-353, Mar. 2006.

[18] M. Kimura, J. Taya, and A. Nakashima, "Temperature dependences of $\mathrm{I}-\mathrm{V}$ characteristics of SD and LDD poly-Si TFTs," IEEE Electron. Device Lett., vol. 33, no. 5, pp. 682-684, May 2012.

[19] J. K. Jeong, "The status and perspectives of metal oxide thin-film transistors for active matrix flexible displays," Semicond. Sci. Technol., vol. 26, no. 3, Mar. 2011.

[20] K. Takechi et al., "Temperature-dependent transfer characteristics of amorphous InGaZnO4 thin-film transistors," Japanese J. Appl Phys., vol. 48, no. 1, Jan. 2009, Art. no. 011301.

[21] H. Godo et al., "Temperature dependence of transistor characteristics and electronic structure for amorphous In-Ga-Zn-oxide thin film transistor," Japanese J. Appl. Phys., vol. 49, no. 3, 2010, Art. no. 03CB04.

[22] "DICOM PS3.14 2016b-Grayscale standard display function," 2016. [Online]. Available: http://dicom.nema.org/medical/dicom/current/ output/pdf/part14.pdf

[23] E. Samei et al., "Assessment of display performance for medical imaging systems: Executive summary of AAPM TG18 report," Med. Phys., vol. 32, no. 4, pp. 1205-1225, 2005.

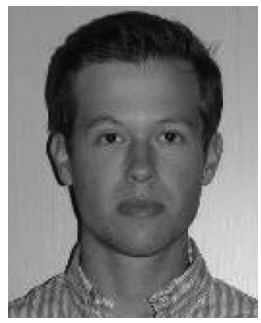

Frédérique Chesterman received the B.S. and the M.S. degrees both in electrical engineering from Ghent University, Ghent, Belgium, in 2011 and 2013, respectively.

$\mathrm{He}$ is currently working toward the Ph.D. degree in photonics engineering in the Department of Electronics and Information Systems, Faculty of Engineering Sciences and Architecture, Ghent University. Since 2013, he has been active as a Research Engineer in the Technology and Innovation Group, Barco Healthcare, Kortrijk, Belgium. His research interests include characterization and modeling of OLED displays and development of compensation algorithms, with specific focus on the medical display market.

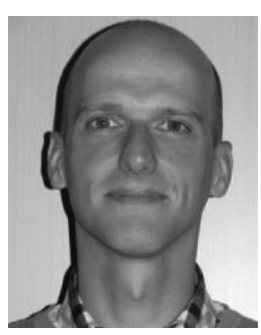

rtrijk, Belgium.
Bastian Piepers received the M.S. degree in electrical engineering from Katholieke Universiteit, Leuven, Belgium, in 2001.

After his graduation he joined as a Hardware Engineer in the Philips Innovative Applications, developing high-end television sets. Afterward, he was with Televic where he first participated in multiple research projects and later became the Architect and Project Lead for the development of nurse call systems. He is currently the Project Manager of the Technology and Innovation Group, Barco Healthcare, Ko- 


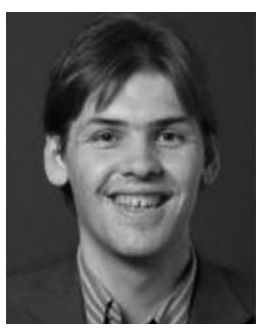

Tom Kimpe received the M.S. degree in computer engineering from Ghent University, Belgium, in 2001, and the $\mathrm{PhD}$ degree in electrical engineering from the same university on the topic of image quality of medical displays. He received the MBA degree from the Vlerick Management School in 2010.

Since 2001, he has been working in Barco's Healthcare Division, where he has been a Development Engineer, Project Manager, Innovation Manager, VP of Technology and Innovation, and Chief Technology Officer. He is currently coordinating all research and innovation activities of Barco's Healthcare Division worldwide and is steering an international team of engineers in USA, Belgium, and Italy. His main topics of expertise are (medical) display technology, image and signal processing, image quality modeling and human perception, network technology, and medical regulatory aspects. Within these fields he is an acknowledged international expert.

Dr. Kimpe is a member of several program committees (e.g., SPIE medical imaging, SID), and reviewer for various journals. He was also one of the organizers of the MIPS2015 Medical Imaging Perception Society Conference.

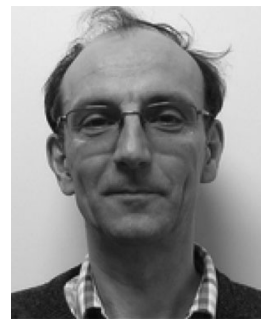

Patrick De Visschere received the degree in electrical engineering in 1976 and the Doctoral degree in applied sciences in 1981, both from Ghent University, Ghent, Belgium.

In 1976, he joined the Department of Electronics and Information Systems, Ghent University, where he has been working on thin-film solar cells. From 1984 to 2000 , he was affiliated with IMEC, and did research on thin-film electroluminescence. He became a parttime and full-time Associate Professor of physical electronics and visualization technology with Ghent University, in 1993 and 2000, respectively. His research interests include physical electronics and optics, electromagnetism, and ray-tracing.

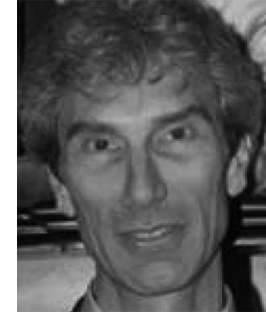

Kristiaan Neyts received the Ph.D. degree from Ghent University, Ghent, Belgium, in thin film electroluminescence in 1992 .

From 1997 to 1998, he was a Postdoc with UC Berkeley. He is currently a Full Research Professor in the Department of Electronics and Information Systems, Faculty of Engineering Sciences and $\mathrm{Ar}$ chitecture, Ghent University. Now, he is also heading the Liquid Crystals and Photonics Group, which is conducting research in the fields of liquid crystals, OLEDs, electrophoresis, and microscopy. This group of 20 researchers has expertise in device technology, numerical simulations, and electro-optical characterization of photonic components. Since 2004, he has been the promoter of 18 completed Ph.D.s. 